\title{
On a property of weak resolvents and its application to a spectral problem
}

\author{
by Yoichi Uetake (Poznań)
}

\begin{abstract}
We show that the poles of a resolvent coincide with the poles of its weak resolvent up to their orders, for operators on Hilbert space which have some cyclic properties. Using this, we show that a theorem similar to the Mlak theorem holds under milder conditions, if a given operator and its adjoint have cyclic vectors.
\end{abstract}

1. Introduction. For a linear bounded operator $A: X \rightarrow X$, where $X$ is a Hilbert space, we define a complex-valued function $\varphi(z)=\langle c,(z I-$ $\left.A)^{-1} b\right\rangle$, which we call a weak resolvent, due to Fong, Nordgren, Radjavi, and Rosenthal (cf. [3], [15]). Here $b, c \in X$, and $\langle f, g\rangle$ denotes the scalar product of the vectors $f$ and $g$. Nordgren et al. considered this function in the study of the invariant subspace problem. Earlier, in the 1960's, in the model theory of operators, Sz.-Nagy and Foias introduced this kind of functions (cf. [17]). Also, in the study of the spectral problem, Mlak proved the following theorem, which also concerns model theory. See also Lebow [12] and Nikol'skiı [14].

Theorem 1 ([13]). If, for every $b, c$ in $X, z^{-1} \varphi\left(z^{-1}\right)=\left\langle c,(I-z A)^{-1} b\right\rangle$ $\in H^{1}$, then $\varrho(A)<1$. Here $\varrho(A)$ is the spectral radius of $A$.

Janas [7] and Jakóbczak and Janas [6] have extended the above theorem to several commuting operators.

During the 1960's, Lax and Phillips developed a scattering theory (cf. [11]). Meanwhile, during the same period, engineers developed independently a control theory, initiated by, among others, Kalman (cf. [9], [8]). Surprisingly enough, the above kind of abstract operator theory and these two theories have been shown to be related to one another by Adamyan and Arov (see references in [11]) and Helton ([4], [5]). The weak resolvent cor-

1991 Mathematics Subject Classification: 47A10, 47A45, 47A40, 30D55, 93B.

Key words and phrases: weak resolvent, cyclic vector, spectral radius, Hardy class, operator model theory, scattering theory, control theory. 
responds to a scattering matrix in scattering theory and a transfer function (or a frequency response function) in control theory, respectively. The above Mlak theorem is also related to the input-output stability of control systems (cf. [8]).

In our paper, we show that if both $A$ and its adjoint have cyclic vectors, then the poles of the resolvent of $A$ and their orders exactly coincide with those of the weak resolvent of $A$. Next, using this result, we show that such operators, a result similar to Mlak's theorem holds under milder conditions.

Notations which we use are as follows:

$\mathbb{D}=\{z:|z|<1\}$ (open unit disc in the complex plane),

$\overline{\mathbb{D}}=\{z:|z| \leq 1\}$ (closed unit disc in the complex plane),

$\mathbb{T}=\{z:|z|=1\}$ (unit circle in the complex plane),

$H^{1}=\left\{f(z)\right.$ analytic in $\left.\mathbb{D}: \sup _{r<1} \int_{0}^{2 \pi}\left|f\left(r e^{i \theta}\right)\right| d \theta=\|f\|_{1}<+\infty\right\}$ (Hardy space with $p=1$ ).

\section{Main theorems}

Definition. We say that $b$ is cyclic for $A$ iff $\overline{\bigcup_{n=0}^{\infty} \operatorname{Span}_{k=0}^{n}\left\{A^{k} b\right\}}=X$.

The following lemma is known as the Popov-Belevich-HautusRosenbrock test in control theory for the finite-dimensional case (cf. [8]). To make the paper self-contained, we include the result with a proof for the infinite-dimensional case.

Lemma 1. If $b$ is cyclic for $A$ and $x$ is an eigenvector of $A^{*}$, i.e., for some $z_{0} \in \mathbb{C}, A^{*} x=z_{0} x, x \neq 0$, then $\langle b, x\rangle \neq 0$.

Pr o of. Suppose $\langle b, x\rangle=0$. Then

$$
\begin{aligned}
\langle A b, x\rangle & =\left\langle b, A^{*} x\right\rangle=z_{0}\langle b, x\rangle=0, \\
\left\langle A^{2} b, x\right\rangle & =\left\langle A b, A^{*} x\right\rangle=z_{0}\langle A b, x\rangle=0, \\
& \vdots \\
\left\langle A^{k} b, x\right\rangle & =\left\langle A^{k-1} b, A^{*} x\right\rangle=z_{0}\left\langle A^{k-1} b, x\right\rangle=0 \quad(k=1,2, \ldots),
\end{aligned}
$$

Thus $x \notin \overline{\bigcup_{n=0}^{\infty} \operatorname{Span}_{k=0}^{n}\left\{A^{k} b\right\}}=X$. However, this contradicts the assumption that $b$ is cyclic for $A$. This completes the proof.

In the following theorem and its proof, a pole is an isolated (not accumulating) pole.

THEOREM 2. Let $(z I-A)^{-1}$ be meromorphic in an open neighborhood of $z_{0}$. Further, let $b$ be cyclic for $A$ and $c$ be cyclic for $A^{*}$. Then the weak resolvent $\varphi(z)=\left\langle c,(z I-A)^{-1} b\right\rangle$ has a pole of order $m$ at $z=z_{0}$ if and only if the resolvent $(z I-A)^{-1}$ has a pole of order $m$ at $z=z_{0}$. 
Proof. "If". Write $(z I-A)^{-1}$ in the following form:

$$
\begin{aligned}
(z I-A)^{-1}= & B_{-m}\left(z-z_{0}\right)^{-m}+B_{-(m-1)}\left(z-z_{0}\right)^{-(m-1)}+\ldots+B_{0} \\
& +B_{1}\left(z-z_{0}\right)+\ldots
\end{aligned}
$$

From this,

$$
\left(z_{0} I-A\right) B_{-m}=0 .
$$

Suppose $B_{-m} b \neq 0$. Then, since $A^{*}$ is cyclic for $c$ by assumption, we have $\left\langle c, B_{-m} b\right\rangle \neq 0$ by Lemma 1 , and thus the weak resolvent $\varphi(z)$ has a pole of order $m$ at $z=z_{0}$. Now we show $B_{-m} b \neq 0$. Define the Riesz projection $E\left(z_{0}\right): X \rightarrow X$ by

$$
E\left(z_{0}\right)=\frac{1}{2 \pi i} \oint_{C}(z I-A)^{-1} d z
$$

where $C$, the path of integration, is a small circle about $z_{0}$ containing no other spectral point of $A$. As is well known, $E\left(z_{0}\right)$ is a projection from $X$ onto $X\left(z_{0}\right)=E\left(z_{0}\right) X$ and $A$ commutes with $E\left(z_{0}\right)$. For the spectral theory used in this proof see, e.g., [1], [2], [10]. For each $n=0,1,2, \ldots$ and $z \in \mathbb{C}$ define a subspace $R(z ; n)$ of $X$ by

$$
R(z ; n)=\left\{x:(z I-A)^{n} x=0\right\} .
$$

For each $z \in \mathbb{C}$ define the index $v(z)$ to be the least integer such that $R(z ; v(z))=R(z ; v(z)+1)$. Then obviously

$$
\{0\}=R(z ; 0) \varsubsetneqq R(z ; 1) \varsubsetneqq \ldots \varsubsetneqq R(z ; v(z))=R(z ; v(z)+1) .
$$

From this we see that

$$
\operatorname{dim} R\left(z_{0} ; v\left(z_{0}\right)\right) \geq v\left(z_{0}\right) .
$$

It is known that $X\left(z_{0}\right)=R\left(z_{0}, v\left(z_{0}\right)\right.$ and that if $z_{0}$ is a pole of $(z I-A)^{-1}$ of order $m$ then $v\left(z_{0}\right)=m$. Therefore

$$
\operatorname{dim} X\left(z_{0}\right) \geq m \text {. }
$$

It is also known that

$$
B_{-k}=\left(A-z_{0} I\right)^{k-1} E\left(z_{0}\right), \quad k \geq 1 .
$$

Recalling that $A$ commutes with $E\left(z_{0}\right)$, we get

$$
B_{-m} b=\left(A-z_{0} I\right)^{m-1} E\left(z_{0}\right) b=E\left(z_{0}\right)\left(A-z_{0} I\right)^{m-1} b .
$$

If $B_{-m} b=0$, then $E\left(z_{0}\right) A^{m-1} b$ is a linear combination of $E\left(z_{0}\right) b, E\left(z_{0}\right) A b$, $\ldots, E\left(z_{0}\right) A^{m-2} b$ and thus so is $E\left(z_{0}\right) A^{k} b$ for $k \geq m$. Since $b$ is cyclic for $A$,

$$
\operatorname{dim} X\left(z_{0}\right)=\operatorname{dim} E\left(z_{0}\right) X=\operatorname{dim} E\left(z_{0}\right) \bigcup_{n=0}^{\infty} \operatorname{Span}_{k=0}^{n}\left\{A^{k} b\right\}=X \leq m-1 .
$$

However, this contradicts the fact that $\operatorname{dim} X\left(z_{0}\right) \geq m$. 
"Only if". Let

$$
(z I-A)^{-1}=B_{-k}\left(z-z_{0}\right)^{-k}+B_{-(k-1)}\left(z-z_{0}\right)^{-(k-1)}+\ldots
$$

Since

$$
\begin{aligned}
\varphi(z) & =\left\langle c,(z I-A)^{-1} b\right\rangle \\
& =\left\langle c, B_{-k} b\right\rangle\left(z-z_{0}\right)^{-k}+\left\langle c, B_{-(k-1)} b\right\rangle\left(z-z_{0}\right)^{-(k-1)}+\ldots,
\end{aligned}
$$

$k$ must be $\geq m$. Now suppose $k \geq m+1$. Then by the previous discussion deriving the "if" part, $\varphi(z)$ has a pole $z_{0}$ of order $k$, which is greater than $m$. However, this contradicts the fact that $\varphi(z)$ has a pole $z_{0}$ of order $m$. Thus $k$ must be equal to $m$.

R e m a r k. An analogous theorem holds for finite-dimensional linear systems (cf. [8]). In [5], Helton has proved an analogous theorem for the infinitedimensional continuous time case by embedding a (continuously controllable and observable) system into a Lax-Phillips scattering model and using the result of the Lax-Phillips scattering theory.

We now show a result similar to Mlak's theorem (Theorem 3 below). We need the following lemma.

LEMma 2. If $h(z) \in H^{1}$ and is meromorphic in an open set including $\overline{\mathbb{D}}$, then $h(z)$ has no pole in $\overline{\mathbb{D}}$.

Proof. By the definition of $H^{1}$ we see that $h(z) \in H^{1}$ is analytic in $\mathbb{D}$ and thus has no pole in $\mathbb{D}$. So we prove that $h(z)$ has no pole on $\mathbb{T}$. A function $h(z)$ that is meromorphic in an open set containing $\mathbb{T}$ may have only a finite number of poles on $\mathbb{T}$, since if there exist an infinite number of poles on $\mathbb{T}$, then there exists an accumulating point on $\mathbb{T}$ since $\mathbb{T}$ is compact. However, this contradicts the definition of meromorphic functions (see, e.g., [16]). Let the finite number of poles of $h(z)$ on $\mathbb{T}$ (and thus in $\overline{\mathbb{D}}$ ) be $z_{1}, \ldots, z_{m}$. Then at each point $z_{i}, i=1, \ldots, m, h(z)$ can be written locally in the form

$$
h(z)=\eta_{i}(z)+\psi_{i}(z),
$$

where

$$
\eta_{i}(z)=b_{i n_{i}}\left(z-z_{i}\right)^{-n_{i}}+\ldots+b_{i 1}\left(z-z_{i}\right)^{-1}, \quad b_{i n_{i}} \neq 0, i=1, \ldots, m,
$$

and $\psi_{i}(z)$ is analytic in a neighbourhood of $z_{i}$. Since $\eta_{i}(z), i=1, \ldots, m$, is analytic in $\overline{\mathbb{D}}-\left\{z_{i}\right\}$, it is easily seen that

$$
\psi(z)=h(z)-\sum_{i=1}^{m} \eta_{i}(z)
$$

is analytic in $\overline{\mathbb{D}}$. Thus $h(z)$ can be written as

$$
h(z)=\psi(z)+\sum_{i=1}^{m} \eta_{i}(z) .
$$


Multiplying $h(z)$ by

$$
q(z)=\left(z-z_{1}\right)^{n_{1}-1}\left(z-z_{2}\right)^{n_{2}} \ldots\left(z-z_{m}\right)^{n_{m}}=\left(z-z_{1}\right)^{n_{1}-1} g(z),
$$

and noting that $g\left(z_{1}\right) \neq 0$, we obtain

$$
q(z) h(z)=\frac{c}{z-z_{1}}+p(z)+q(z) \psi(z)
$$

where $p(z)$ is a polynomial and $c \neq 0$. Since $p(z), q(z)$ and $\psi(z)$ are holomorphic in $\overline{\mathbb{D}}$ and thus bounded in $\overline{\mathbb{D}}$, if $h(z) \in H^{1}$ then

$$
\begin{aligned}
\sup _{r<1} \int_{0}^{2 \pi} & \left|\frac{c}{r e^{i \theta}-z_{1}}\right| d \theta \\
& \leq \sup _{r<1}^{2 \pi} \int_{0}^{2 \pi}\left[\left|q\left(r e^{i \theta}\right)\right| \cdot\left|h\left(r e^{i \theta}\right)\right|+\left|p\left(r e^{i \theta}\right)\right|+\left|q\left(r e^{i \theta}\right)\right| \cdot\left|\psi\left(r e^{i \theta}\right)\right|\right] d \theta \\
& <\infty .
\end{aligned}
$$

Thus $c /\left(z-z_{1}\right) \in H^{1}$. However, this is obviously impossible, which follows immediately from the celebrated Hardy inequality, i.e.,

$$
\sum_{n=1}^{\infty} \frac{\left|a_{n}\right|}{n+1} \leq \frac{1}{\pi}\|f\|_{1}
$$

for $f(z)=a_{0}+a_{1} z+a_{2} z^{2}+\ldots \in H^{1}$. Therefore $h(z)$ has no pole in $\overline{\mathbb{D}}$.

We can now prove the following theorem.

Theorem 3. Assume that $(I-z A)^{-1}$ is meromorphic in an open set including $\overline{\mathbb{D}}$. Let $b$ be cyclic for $A$ and $c$ be cyclic for $A^{*}$. If $z^{-1} \varphi\left(z^{-1}\right)=$ $\left\langle c,(I-z A)^{-1} b\right\rangle \in H^{1}$, then $\varrho(A)<1$.

Proof. By the assumption and Lemma $2, z^{-1} \varphi\left(z^{-1}\right)$ has no pole in $\overline{\mathbb{D}}$. Thus $\varphi(z)=\left\langle c,(z I-A)^{-1} b\right\rangle$ has no pole in $\mathbb{C}-\mathbb{D}=\{z:|z| \geq 1\}$. Hence, by Theorem $2,(z I-A)^{-1}$ has no pole in $\mathbb{C}-\mathbb{D}$. Therefore $\varrho(A) \leq 1$. Now suppose $\varrho(A)=1$. Then there exists an infinite sequence $z_{1}, z_{2}, \ldots$ in $\sigma(A)$, the spectrum of $A$, such that $\left|z_{i}\right| \rightarrow 1$. Since the spectrum of $A$ is compact, a subsequence of $\left\{z_{i}\right\}$ has a limit point $z_{0} \in \sigma(A)$ on $\mathbb{T}$. However, again by Lemma 2 and Theorem 2, this is impossible. Thus $\varrho(A)<1$.

Acknowledgements. I would like to thank Prof. Jaroslav Zemánek for encouragement, stimulating discussion and helpful comments. I would like to thank Prof. Jan Janas for helpful suggestions. I would like to thank Prof. Krzysztof Rudol for improving the proof of Lemma 2. I would also like to thank Prof. Olavi Nevanlinna for correcting some points of the original manuscript. Finally, I would like to thank Prof. Graham R. Allan, Prof. Joseph Ball and Prof. J. A. van Casteren for helpful comments. 


\section{References}

[1] H. R. Dowson, Spectral Theory of Linear Operators, Academic Press, London, 1978.

[2] N. Dunford and J. T. Schwartz, Linear Operators, Part I: General Theory, Intescience, New York, 1958.

[3] C. K. Fong, E. A. Nordgren, H. Radjavi and P. Rosenthal, Weak resolvents of linear operators, II, Indiana Univ. Math. J. 39 (1990), 67-83.

[4] J. W. Helton, Discrete time systems, operator models, and scattering theory, J. Funct. Anal. 16 (1974), 15-38.

[5] - Systems with infinite-dimensional state space: the Hilbert space approach, Proc. IEEE 64 (1976), 145-160.

[6] P. Jakóbczak and J. Janas, On Nikolski theorem for several operators, Bull. Polish Acad. Sci. Math. 31 (1983), 369-374.

[7] J. Janas, On a theorem of Lebow and Mlak for several commuting operators, Studia Math. 76 (1983), 249-253.

[8] T. Kailath, Linear Systems, Prentice-Hall, Englewood Cliffs, N.J., 1980.

[9] R. E. Kalman, P. L. Falb and M. A. Arbib, Topics in Mathematical System Theory, McGraw-Hill, New York, 1969.

[10] T. Kato, Perturbation Theory for Linear Operators, 2nd ed., Springer, Berlin, 1976.

[11] P. D. Lax and R. S. Phillips, Scattering Theory, rev. ed., Academic Press, New York, 1989.

[12] A. Lebow, Spectral radius of an absolutely continuous operator, Proc. Amer. Math. Soc. 36 (1972), 511-514.

[13] W. Mlak, On a theorem of Lebow, Ann. Polon. Math. 35 (1977), 107-109.

[14] N. K. Nikol'skiur, A Tauberian theorem on the spectral radius, Sibirsk. Mat. Zh. 18 (1977), 1367-1372 (in Russian).

[15] E. Nordgren, H. Radjavi and P. Rosenthal, Weak resolvents of linear operators, Indiana Univ. Math. J. 36 (1987), 913-934.

[15] W. Rudin, Real and Complex Analysis, 2nd ed., McGraw-Hill, New York, 1974.

[16] B. Sz.-Nagy and C. Foiaş, Harmonic Analysis of Operators on Hilbert Space, North-Holland, Amsterdam, 1970.

Department of Mathematics and Computer Science

Adam Mickiewicz University

Matejki 48/49

60-769 Poznań, Poland

E-mail: uetake@math.amu.edu.pl 